\title{
Radiometric Modeling and Calibration of the Geostationary Imaging Fourier Transform Spectrometer (GIFTS) Ground Based Measurement Experiment
}

\author{
Jialin $\operatorname{Tian}^{a}$, William L. Smith ${ }^{b}$, and Michael J. Gazarik ${ }^{a}$ \\ ${ }^{a}$ NASA Langley Research Center, Hampton, VA 23681-2199; \\ ${ }^{b}$ Hampton University/University of Wisconsin-Madison, Hampton, VA 23668
}

\begin{abstract}
The ultimate remote sensing benefits of the high resolution Infrared radiance spectrometers will be realized with their geostationary satellite implementation in the form of imaging spectrometers. This will enable dynamic features of the atmosphere's thermodynamic fields and pollutant and greenhouse gas constituents to be observed for revolutionary improvements in weather forecasts and more accurate air quality and climate predictions. As an important step toward realizing this application objective, the Geostationary Imaging Fourier Transform Spectrometer (GIFTS) Engineering Demonstration Unit (EDU) was successfully developed under the NASA New Millennium Program, 2000-2006. The GIFTS-EDU instrument employs three focal plane arrays (FPAs), which gather measurements across the long-wave IR (LWIR), short/mid-wave IR (SMWIR), and visible spectral bands. The GIFTS calibration is achieved using internal blackbody calibration references at ambient (260 $\mathrm{K})$ and hot $(286 \mathrm{~K})$ temperatures. In this paper, we introduce a refined calibration technique that utilizes Principle Component (PC) analysis to compensate for instrument distortions and artifacts, therefore, enhancing the absolute calibration accuracy. This method is applied to data collected during the GIFTS Ground Based Measurement (GBM) experiment, together with simultaneous observations by the accurately calibrated AERI (Atmospheric Emitted Radiance Interferometer), both simultaneously zenith viewing the sky through the same external scene mirror at ten-minute intervals throughout a cloudless day at Logan Utah on September 13, 2006. The accurately calibrated GIFTS radiances are produced using the first four PC scores in the GIFTS-AERI regression model. Temperature and moisture profiles retrieved from the PC-calibrated GIFTS radiances are verified against radiosonde measurements collected throughout the GIFTS sky measurement period. Using the GIFTS GBM calibration model, we compute the calibrated radiances from data collected during the moon tracking and viewing experiment events. From which, we derive the lunar surface temperature and emissivity associated with the moon viewing measurements.
\end{abstract}

Keywords: Geostationary Imaging Fourier Transform Spectrometer (GIFTS); imaging Fourier transform spectrometer; radiometric calibration; Principle Component (PC) analysis; remote sensing, lunar surface temperature, lunar emissivity.

\section{INTRODUCTION}

The implementation of Infrared ultra-spectral resolution spectrometers on polar orbiting satellites has ushered in a new era in satellite remote atmospheric sounding capability. However, the ultimate benefits of the ultra-spectral remote sensing technology are expected to be realized through its geostationary satellite implementation in the form of imaging spectrometers. This will enable dynamic features of the atmosphere's thermodynamic fields and pollutant and greenhouse gas constituents to be observed for revolutionary improvements in weather forecasts and more accurate air quality and climate predictions. As an important step toward realizing this application objective, the Geostationary Imaging Fourier Transform Spectrometer (GIFTS) Engineering Demonstration Unit (EDU) was successfully developed under the NASA New Millennium Program, 2000-2006. The GIFTS-EDU instrument employs three focal plane arrays (FPAs), which gather measurements across the long-wave (685$1130 \mathrm{~cm}^{-1}$ ) IR (LWIR), short/mid-wave (1650-2250 $\mathrm{cm}^{-1}$ ) IR (SMWIR), and visible spectral bands. The raw

Further author information: (Send correspondence to Jialin Tian) Jialin Tian: E-mail: Jialin.Tian-1@nasa.gov, Telephone: 757-864-3698 
interferogram measurements are radiometrically and spectrally calibrated to produce radiance spectra, which are further processed to obtain atmospheric profiles via retrieval algorithms. The absolute radiometric performance of the instrument is affected by several factors including the FPA off-axis effect, detector/readout electronics induced nonlinearity distortions, and fore-optics offsets. The GIFTS-EDU, being the very first imaging spectrometer to use ultra-high speed electronics to readout its large area format focal plane array detectors, operating at wavelengths as large as 15 microns, possessed non-linearity's not easily removable in the initial calibration process. In this paper, we introduce a refined calibration technique that utilizes Principle Component (PC) analysis to compensate for instrument distortions remaining after the initial calibration procedures. In this paper, we emphasize the technique of $\mathrm{PC}$ regression calibration and demonstrate the sensitivity of the calibrated radiances to atmospheric temperature and moisture variability using data collected with the GIFTS and the AERI, both simultaneously zenith viewing the sky through the same external scene mirror at ten-minute intervals throughout a cloudless day at Logan Utah on September 13, 2006. In Section 2, we introduce the methodology and results of the $\mathrm{PC}$ regression calibration technique. In Section 3, we present the temperature and moisture retrieval method using the PC calibrated radiances. And in Section 4, we apply the same calibration principle to data collected during the moon viewing events. From which, we compute the lunar surface temperature and emissivity.

\section{GIFTS CALIBRATIONS USING PRINCIPLE COMPONENT REGRESSION}

In this section, we introduce a refined calibration technique that utilizes principle component analysis to compensate for instrument distortions and artifacts remaining after the initial radiometric calibration process. This method is applied to data collected with the GIFTS, together with simultaneous observations by the accurately calibrated AERI (Atmospheric Emitted Radiance Interferometer), both zenith viewing the sky at ten-minute intervals throughout a cloudless day of an atmospheric measurement experiment. The PC vectors of the calibrated radiance spectra are defined from the AERI observations and regression matrices relating the initial GIFTS radiance $\mathrm{PC}$ scores to the AERI radiance PC scores are calculated using the least squares inverse method. A new set of accurately calibrated GIFTS radiances are produced using the first four PC scores in the regression model. The PC-calibrated radiances and initial calibration results are compared to AERI spectra in terms of FPA mean and variance radiance differences.

\subsection{GIFTS and AERI Data Definitions}

We define $G(n, k, x, y, s)$ as calibrated GIFTS GBM AVE (Atmospheric Variation Event) long-wave radiance spectra, where $k=1, \ldots, K$ is the spectral sample number and $K=778$ is the total number of spectral samples. The corresponding spectral wavenumber is written as $\sigma_{k}=\sigma_{k_{0}}+k \Delta \sigma$, where $\Delta \sigma=0.5733 \mathrm{~cm}^{-1}$ is spectral resolution and $\sigma_{k}$ covers from $684.56 \mathrm{~cm}^{-1}$ to $1130.04 \mathrm{~cm}^{-1}$. The variable $n=1, \ldots, N$ is the temporal sample number and $N=52$ is the total number of temporal samples; i.e., $t=t_{0}+n \Delta t$, and $\Delta t=10$ minutes. The variable $s=1, \ldots, S$ is the scan number and $S=5$ is the total number of scans collected during each data measurement period. Finally, $x, y=1, \ldots, 128$ represent the pixel index numbers in rows and columns, respectively. We define $\bar{G}(n, k, x, y)$ as the scan-averaged data cubes, and $\overline{\bar{G}}(k, x, y)$ as the scan-temporal-averaged data cube, and $\mathbf{X}_{G}^{x, y}$ as the "centered" AVE data cube set, in which the scan-temporal-mean is removed from the initial data cube set.

$$
\mathbf{X}_{G}^{x, y}=\bar{G}(n, k, x, y)-\overline{\bar{G}}(k, x, y) .
$$

Similarly, the notation for the accurately calibrated AERI radiances is given as $A(n, k)$. Since AERI has one pixel element, the variables $x, y$ are eliminated from the following expressions.

$$
\begin{aligned}
\bar{A}(k) & =\frac{1}{N} \sum_{n} A(n, k), \quad \text { and } \\
\mathbf{X}_{A} & =A(n, k)-\bar{A}(k) .
\end{aligned}
$$

\subsection{PC Regression Calibration}

The principle component regression model that relates the GIFTS AVE data cube set and AERI data set is

$$
\mathbf{X}_{A}=\mathbf{X}_{G}^{x, y} \mathbf{R}^{x, y}+\mathbf{F},
$$


where $\mathbf{R}^{x, y}$ denotes the GIFTS-AERI regression matrix and $\mathbf{F}$ represents random noise and errors. By using the singular value decomposition (SVD), $\mathbf{X}_{A}$ and $\mathbf{X}_{G}$ can be factorized into

$$
\mathbf{X}_{A}=\mathbf{T}_{A} \mathbf{P}_{A}^{T} \quad \text { and } \quad \mathbf{X}_{G}^{x, y}=\left(\mathbf{T}_{G} \mathbf{P}_{G}^{T}\right)^{x, y}
$$

We define the number of AERI and GIFTS eigenvectors to be apply into the prediction model as variables $a$ and $g$, respectively. The values of $a$ and $g$ are set to be four, which were determined experimentally such that both the individual features of GIFTS pixels and AERI are preserved in the prediction model. The matrices $\mathbf{X}_{A_{a}}$ and $\mathbf{T}_{G_{g}}^{x, y}$ for AERI and GIFTS can be related by $\mathbf{X}_{A_{a}}=\left(\mathbf{T}_{G_{g}} \mathbf{C}\right)^{x, y}$; the matrix $\mathbf{C}^{x, y}$ is estimated by applying the least squares inversion, i.e.,

$$
\widehat{\mathbf{C}}^{x, y}=\left(\left(\mathbf{T}_{G_{g}}^{T} \mathbf{T}_{G_{g}}\right)^{-1} \mathbf{T}_{G_{g}}^{T} \mathbf{X}_{A_{a}}\right)^{x, y} .
$$

Finally, we can predict the GIFTS radiances $\widehat{G}(n, k, x, y)$ using the GIFTS-AERI regression matrix $\mathbf{R}^{x, y}=$ $\mathbf{P}_{G_{g}}^{x, y} \widehat{\mathbf{C}}^{x, y}$ :

$$
\widehat{G}(n, k, x, y)=\bar{A}(k)+[\bar{G}(n, k, x, y)-\overline{\bar{G}}(k, x, y)] \mathbf{P}_{G_{g}}^{x, y} \widehat{\mathbf{C}}^{x, y} .
$$

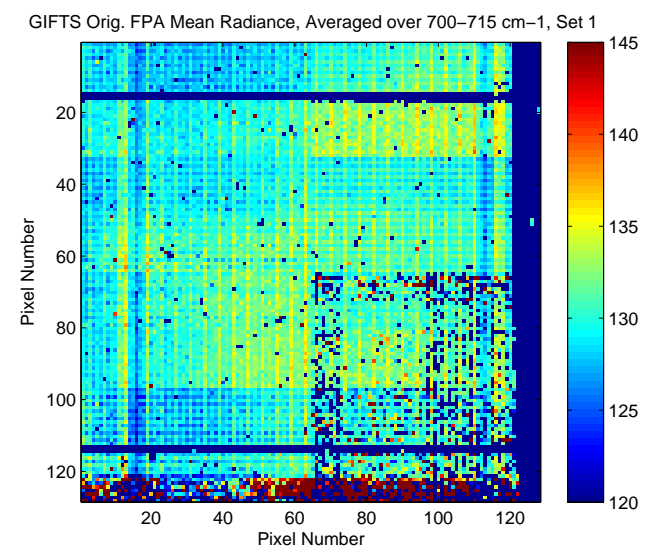

(a)

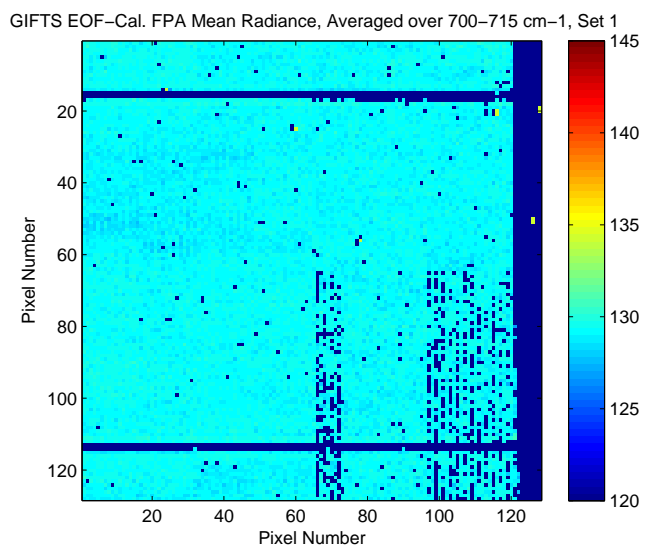

(c)

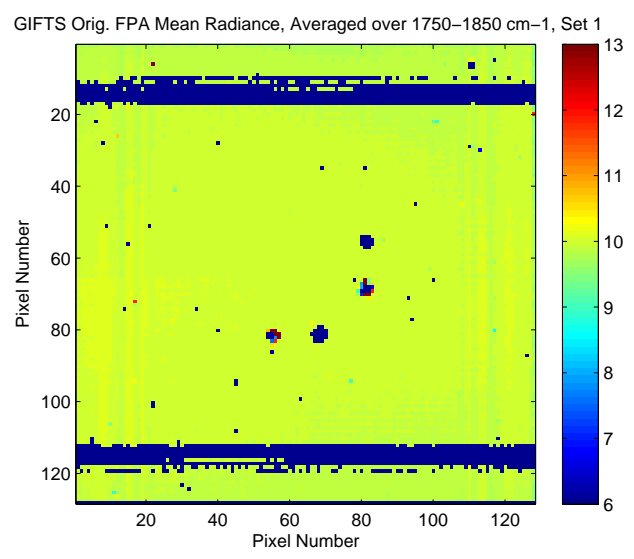

(b)

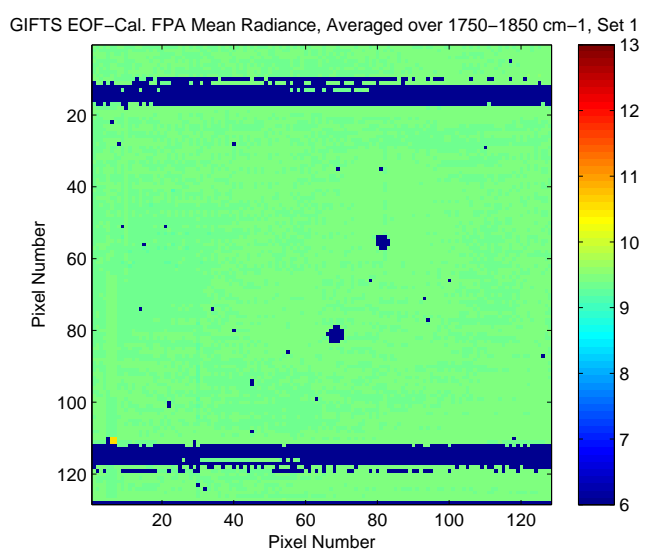

(d)

Figure 1. FPA mean radiance images for temporal sample Set 1 (observed from Logan UT at 5 AM on 13 September, 2006): (a) long-wave radiances averaged over $700-715 \mathrm{~cm}^{-1}$ from GIFTS original calibration, (b) shortwave radiances averaged over 1750-1850 $\mathrm{cm}^{-1}$ from GIFTS original calibration, (c) long-wave radiances averaged over $700-715 \mathrm{~cm}^{-1}$ from GIFTS PC calibration, and (d) shortwave radiances averaged over $1750-1850 \mathrm{~cm}^{-1}$ from GIFTS PC calibration. 
The FPA images for GIFTS initial calibration and PC calibration results can be seen in Figs. 1(a)-(d). These plots indicate that the FPA electronic readout noise has been significantly reduced after applying the PC calibration method. Comparisons between GIFTS calibration results and AERI are made, and the differences in radiance between GIFTS and AERI can be seen in Figs. 2(a)-(d). After performing the PC calibration, the mean and standard deviation values of the differences between GIFTS and AERI radiances have been decreased.

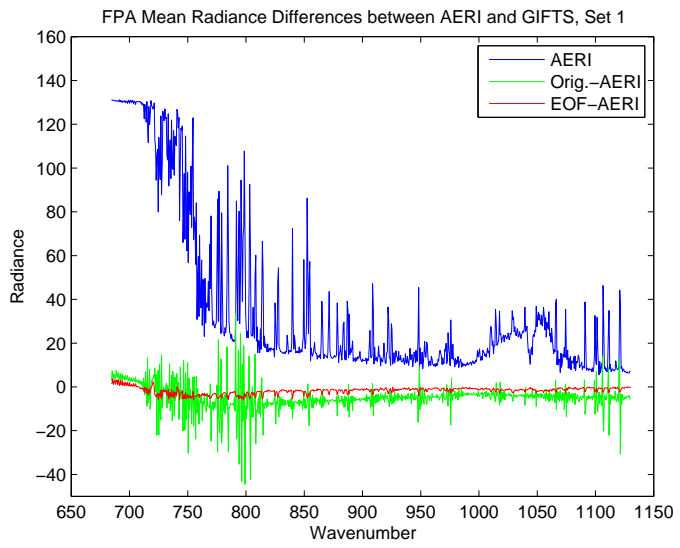

(a)

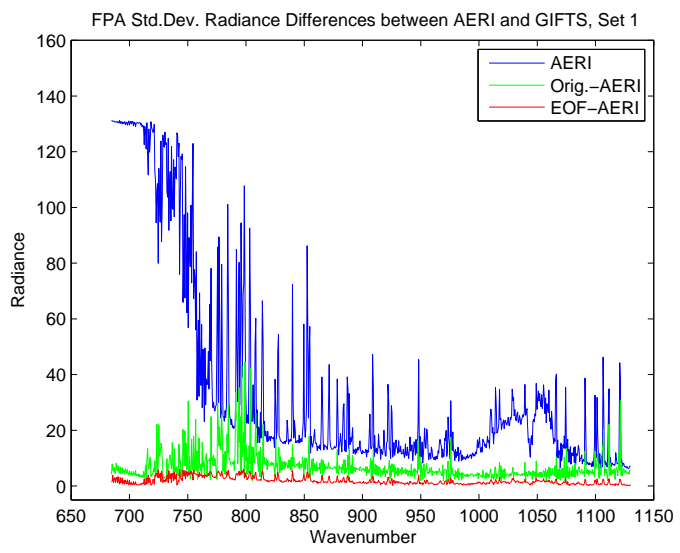

(c)

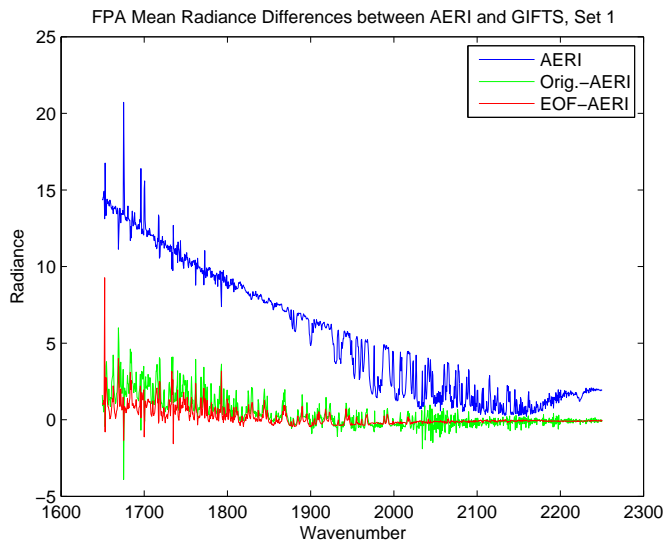

(b)

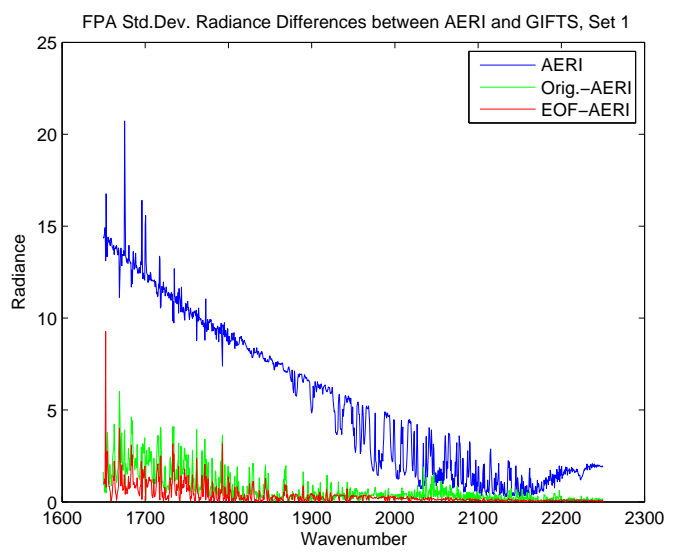

(d)

Figure 2. FPA mean radiance comparisons for temporal sample Set 1 (observed from Logan UT at 5 AM on 13 September, 2006): (a) long-wave FPA mean radiance differences between AERI and GIFTS calibration results, (b) short-wave FPA mean radiance differences between AERI and GIFTS calibration results, (c) long-wave FPA standard deviation of radiance differences between AERI and GIFTS calibration results, and (d) short-wave FPA standard deviation of radiance differences between AERI and GIFTS calibration results.

\section{TEMPERATURE AND MOISTURE RETRIEVAL}

In this section, temperature and moisture profiles are determined using the PC-calibrated upward viewing GIFTS radiances that are obtained through the calibration process described in the previous section. The results are derived and verified using radiosonde measurements collected throughout the GIFTS 9-hour sky viewing measurement period on September 13, 2006. The purpose of the retrieval process was to demonstrate the sensitivity of the calibrated GIFTS measurements to the temporal variability of atmospheric temperature and moisture vertical structure. For the retrieval operation, we use a new set of data cubes that combines both the 
longwave and shortwave spectral bands. Given $l=1, \ldots, L$ as the short-wave spectral sample number, where $L=1049, \sigma_{l}=\sigma_{l_{0}}+l \Delta \sigma$, and $\Delta \sigma=0.5733 \mathrm{~cm}^{-1} ; \sigma_{l}$ covers from $1649.48 \mathrm{~cm}^{-1}$ to $2250.33 \mathrm{~cm}^{-1}$. Define the new GIFTS and AERI data sets as

$$
\begin{aligned}
\mathbf{X}_{G}^{x, y} & =\bar{G}(n, m, x, y)-\overline{\bar{G}}(m, x, y) \quad \text { and } \\
\mathbf{X}_{A} & =A(n, m)-\bar{A}(m),
\end{aligned}
$$

respectively, where $m=[k, l]$ combines the long-wave and short-wave spectral sample numbers. Similar to previous deviations, we can estimate $\widehat{\mathbf{C}}^{x, y}$ from $\mathbf{X}_{G}^{x, y}$ and $\mathbf{X}_{A}$. The mean value of $\widehat{\mathbf{C}}^{x, y}$ averaged over the entire FPA, excluding bad pixels, is computed from

$$
\overline{\widehat{\mathbf{C}}}=\frac{1}{Z} \sum_{(x, y) \in \mathcal{P}} \widehat{\mathbf{C}}^{x, y}
$$

where $\mathcal{P}$ denotes the set contains the good pixels, and $Z$ is the total count of good pixels. From which, we compute

$$
\mathbf{D}^{x, y}=\mathbf{X}_{G}^{x, y} \overline{\widehat{\mathbf{C}}}^{T},
$$

$\mathbf{D}^{x, y}$ contains the matrix of coefficients of the AERI radiance eigenvectors that are predicted from the GIFTS spectra. Given radiosonde measurements of temperature and mixing ratio on the same day as functions of pressure as $T(h, n)$ and $Q(h, n)$, where $n=1, \ldots, N$ and $N=52$. The pressure level is represented by $h=$ $1, \ldots, H$ and $H=31$. The corresponding pressure value is given by $p=p_{0}+h \Delta p ; p$ covers from $1000 \mathrm{mb}$ to $700 \mathrm{mb}$, and $\Delta p=10 \mathrm{mb}$. Define $\bar{T}(h)=\frac{1}{N} \sum_{n} T(h, n), \mathbf{T}=T(h, n)-\bar{T}(h)$ and $\bar{Q}(h)=\frac{1}{N} \sum_{n} Q(h, n), \mathbf{Q}=$ $Q(h, n)-\bar{Q}(h)$, we can establish the regression models for temperature and moisture as $\mathbf{T}^{x, y}=(\mathbf{D A})^{x, y}+\mathbf{F}_{T}$ and $\mathbf{Q}^{x, y}=(\mathbf{D B})^{x, y}+\mathbf{F}_{Q}$. The matrices $\widehat{\mathbf{A}}^{x, y}$ and $\widehat{\mathbf{B}}^{x, y}$ are solved using the least squares inversions:

$$
\begin{aligned}
\widehat{\mathbf{A}}^{x, y} & =\left(\left(\mathbf{D}^{T} \mathbf{D}\right)^{-1} \mathbf{D}^{T} \mathbf{T}\right)^{x, y} \\
\widehat{\mathbf{B}}^{x, y} & =\left(\left(\mathbf{D}^{T} \mathbf{D}\right)^{-1} \mathbf{D}^{T} \mathbf{Q}\right)^{x, y}
\end{aligned}
$$

The temperature and moisture as functions of pressure and time are predicted from

$$
\begin{aligned}
\widehat{\mathbf{T}}^{x, y} & =(\mathbf{D} \widehat{\mathbf{A}})^{x, y}+\bar{T}(h) \\
\widehat{\mathbf{Q}}^{x, y} & =(\mathbf{D} \widehat{\mathbf{B}})^{x, y}+\bar{Q}(h),
\end{aligned}
$$

respectively. Figs. 3(a)-(f) contain time cross-sections, shown as false color images, of temperature and moisture profiles observed by 1.5 hour interval radiosonde measurements, and the 10-minute interval AERI and GIFTS PC-based algorithm retrievals. The 52 10-minute time interval samples cover a time period of nine hours between 5 AM and 2 PM local mountain daylight time at Logan Utah on September 13, 2006. In Figs. 4(a) and 4(b), the linearly interpolated point measurements by the radiosonde during this time period are compared to the AERI and GIFTS retrieved point values for temperature and relative humidity, respectively, at five different atmospheric levels. Comparisons are made based on these three sets of profiles; the results are shown in Figs. 4(a) and 4(b). The very high sensitivity of the GIFTS observations to spatial and temporal variability of temperature and moisture is clearly shown. The results provide confidence that if the GIFTS were to be flown on a geostationary satellite, the remote sensing and forecast application objectives of the GIFTS measurements would be achieved.

\section{LUNAR SURFACE TEMPERATURE AND EMISSIVITY ESTIMATION}

In this section, we discuss the results obtained from the lunar measurements collected during the moon viewing events (MVE). This phase of the GBM experiment is designed to capture high quality lunar images across the GIFTS EDU spectral channels at a scan resolution of $9.18 \mathrm{~cm}^{-1}$. Each MVE data set is consist of multiple scans of the lunar images, which are radiometrically calibrated to produce the MVE radiance spectra. The atmospheric 


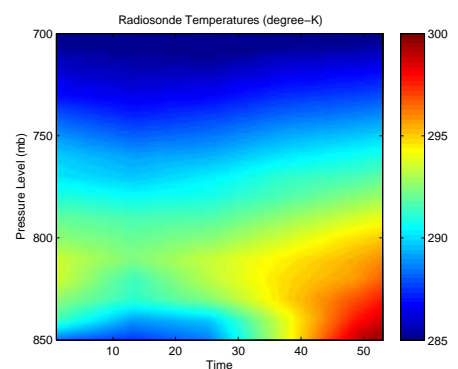

(a)

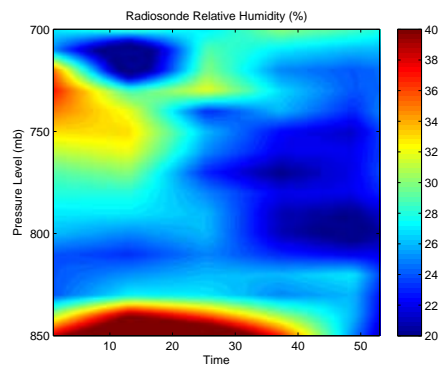

(d)

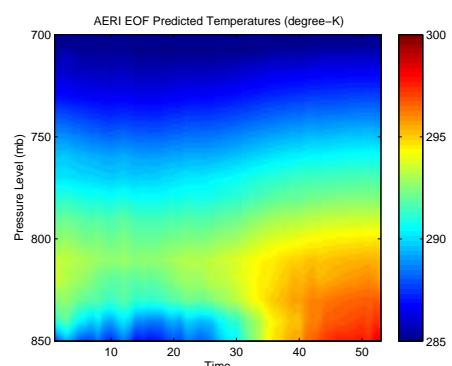

(b)

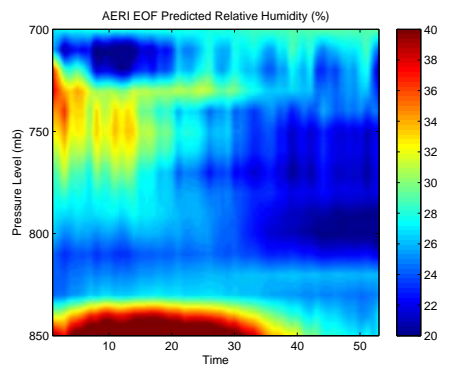

(e)

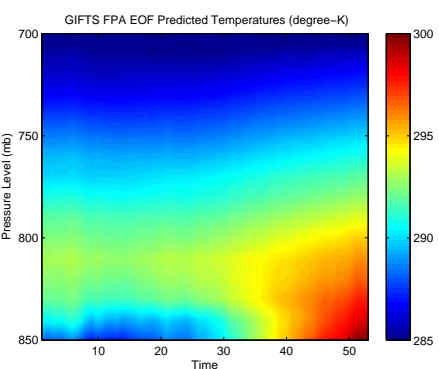

(c)

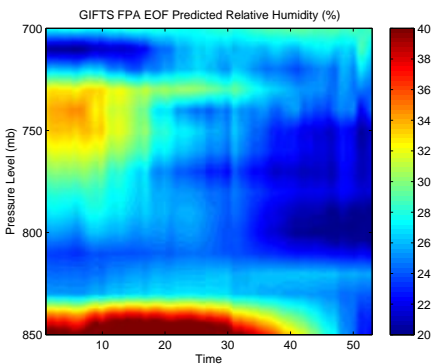

(f)

Figure 3. Vertical cross-sections of temperature and moisture observed at Logan UT between 5 AM and 2 PM MDT on 13 September 2006: (a) radiosonde temperatures, (b) AERI predicted temperatures, (c) GIFTS PC predicted temperatures, (d) radiosonde relative humidity measurements, (e) AERI predicted relative humidity, and (f) GIFT PC predicted relative humidity.

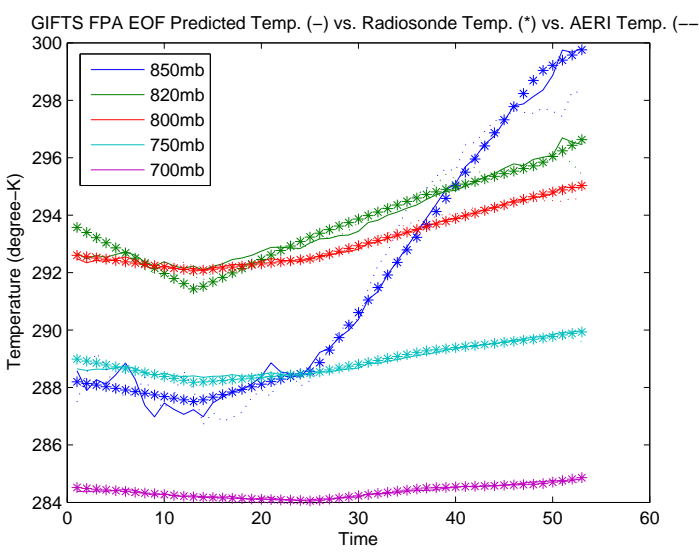

(a)

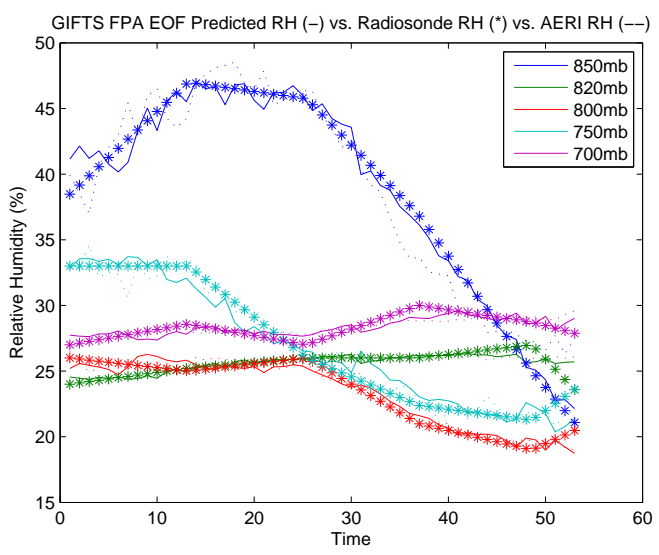

(b)

Figure 4. Comparisons of GIFTS and AERI 10-minute interval retrieved temperature and moisture values to linearly interpolated $1.5 \mathrm{hr}$ interval radiosonde measurements observed at five different atmospheric levels at Logan UT between 5 AM and 2 PM MDT on 13 September 2006: (a) radiosonde temperature measurements vs. GIFTS PC predicted and AERI predicted temperatures, and (b) radiosonde moisture measurements vs. GIFTS PC predicted and AERI predicted relative humidity. 
background effects are then removed from scans that contain lunar images. In the following step, we align the longwave and shortwave scans such that lunar images for simultaneous longwave and shortwave scans are spatially co-registering. Figs. 5(a) and (b) show the MVE radiance spectrum for a single pixel that is located within the lunar image and the mean brightness temperature averaged over $1100.7986-1119.1453 \mathrm{~cm}^{-1}$, respectively. Using the line-by-line calculated atmospheric transmittance, we estimate the lunar surface temperature and emissivity. The results of estimated mean lunar surface temperature and emissivity over $1100.7986-1119.1453 \mathrm{~cm}^{-1} \mathrm{can}$ be seen in Figs. 6(a) and (b), respectively.

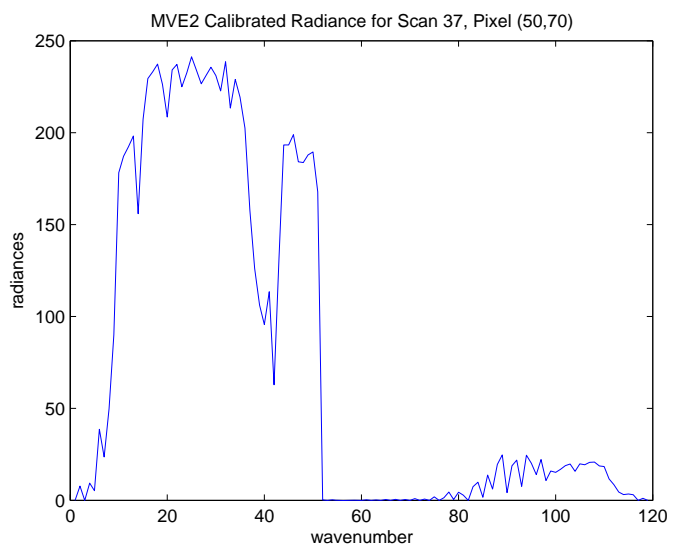

(a)

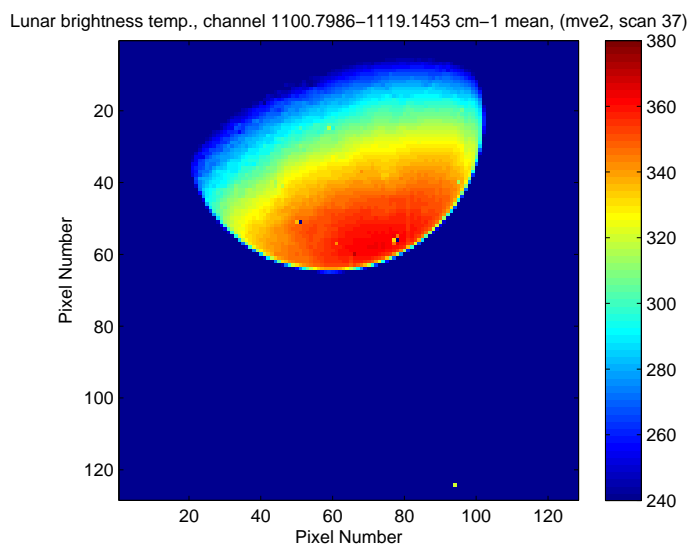

(b)

Figure 5. Longwave and shortwave MVE radiance spectrum and brightness temperature: (a) MVE radiance spectrum for Pixel (50,70), and (b) MVE mean brightness temperature averaged over 1100.7986-1119.1453 $\mathrm{cm}^{-1}$.

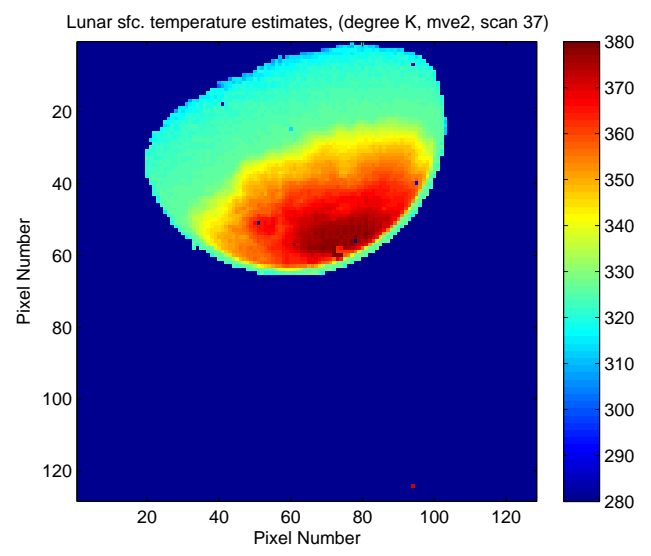

(a)

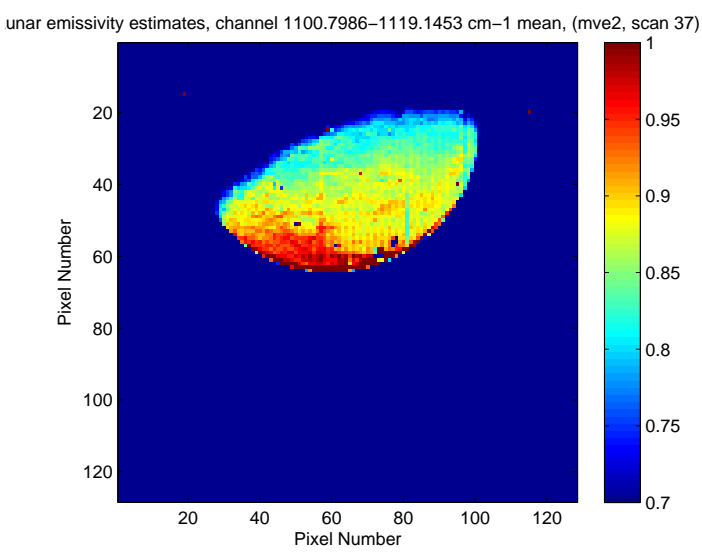

(b)

Figure 6. Estimated lunar surface temperature and emissivity: (a) estimated MVE lunar surface temperature, and (b) estimated MVE emissivity averaged over $1100.7986-1119.1453 \mathrm{~cm}^{-1}$.

\section{CONCLUSION}

This paper describes a calibration refinement technique for the GIFTS-EDU designed to remove non-linearity distortions, found difficult to remove in the initial calibration of the data. This technique utilizes Principle Component (PC) analysis to compensate for artifacts remaining after the initial radiometric calibration process, thus further enhancing the absolute calibration accuracy. The algorithm is applied the data collected with GIFTS, 
together with simultaneous observations by the accurately calibrated AERI (Atmospheric Emitted Radiance Interferometer), during an atmospheric measurement experiment conducted at Logan Utah on September 13, 2006. The algorithmic components of this method and the calibration results are discussed in the paper. Temperature and moisture profiles based on retrievals using the PC-calibrated GIFTS radiances are also presented. The very high sensitivity of the GIFTS observations to spatial and temporal variability of temperature and moisture is shown. Furthermore, using the GIFTS GBM calibration model, we compute the calibrated radiances from data collected during the moon tracking and viewing experiment events. From which, we derive the lunar surface temperature and emissivity associated with the moon viewing measurements. The results provide confidence that if the GIFTS were to be flown on a geostationary satellite, the remote sensing and forecast application objectives of the GIFTS measurements would be achieved. 